Highlights of Astronomy, Vol. 12

International Astronomical Union, 2002

H. Rickman, ed.

\title{
Diffuse Cluster-Wide Radio Halos
}

\author{
Haida Liang \\ Physics Dept., University of Bristol, Tyndall Ave., Bristol BS8 1TL, UK
}

\begin{abstract}
We will discuss the properties and origins of halos and relics including estimates of the cluster magnetic fields, and present results for a few recently discovered halos and relics. The electrons in the suprathermal high energy tail of the thermal gas distribution are likely to provide the seed particles for acceleration through mergers and turbulences to relativistic energies. These relativistic particles are then responsible for the synchrotron emission of the halos.
\end{abstract}

\section{Properties of Radio Halos}

Radio halos are preferentially found in high X-ray luminosity clusters (Giovannini et al. 1999) and a tentative correlation was found between the radio luminosity of halos and X-ray temperature of cluster gas (Liang et al. 2000). Recently, we have observed a number of clusters with relatively low temperature $\left(k T_{x}<7 \mathrm{keV}\right)$ to test if this correlation still holds and whether there is a switch-on temperature for radio halo production. We obtained upper-limits from ATCA observations of the clusters A3158, A3921, and updated the $P_{1.4}-k T_{x}$ plot (Fig. 1) with the addition of A2199 (Kempner \& Sarazin 2000) and A3667 (Ekers private comm.). We also attempted to test the theory that merging is the essential ingredient in halo production, since so far all the known radio halos are detected in non-cooling flow, merging clusters (Giovannini et al. 1999). For this reason, we chose to observe with the ATCA a strong cooling flow cluster (i.e. no merging), RXJ1347-11, with a high temperature (Fig. 1). We were not able to reach a low brightness limit because of the relatively strong central radio source in the cluster. The cluster was also fairly small in angular size which made it even more difficult to separate the central radio source from a clusterwide diffuse emission. These features are common to most cooling flow clusters, which may cause a bias in the result that only non-cooling flows have radio halos. Note that we prefer the $P_{1.4}-k T_{x}$ relation to the $P_{1.4}-L_{x}$ relation because it is free from the Malmquist bias common to luminosity-luminosity relations; furthermore $k T_{x}$ is directly related to the cluster total mass.

On the large scale, radio halos have similar extent and shape as the thermal $\mathrm{X}$-ray emission (e.g. Fig. 1). However, the radio emission corresponds better in detail to the galaxy distribution than the X-ray emission in the case of 1E065756 (Liang et al. 2000) and Coma (Kim et al. 1990). In 1E0657-56 and A2744, the clusters have substructure in both $\mathrm{X}$-ray and galaxy distributions with the X-ray subclumps displaced from the galaxy subclumps (Fig 1). 

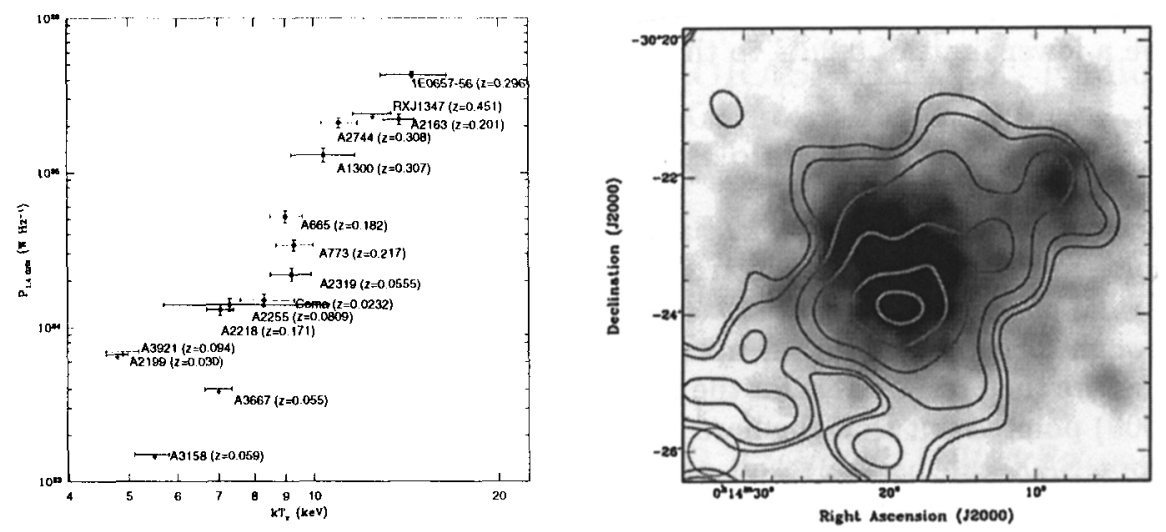

Figure 1. LEFT: Radio power $P_{1.4}$ at $1.4 \mathrm{GHz}$ (rest frame) versus the cluster X-ray temperature $k T_{x}$. Similar to Fig. 9 in Liang et al. (2000) but with the addition of a few upper limits. RIGHT: A grey scale ROSAT PSPC image of A2744 overlaid with contours of $1.3 \mathrm{GHz}$ ATCA image of the radio halo.

\section{Intracluster Magnetic field}

Under the assumption of equipartition, cluster $B$-fields are estimated to be of order of a few $\mu \mathrm{G}$ from the radio brightness of the cluster-wide halos. The first direct measurement of a cluster $B$-field was in the Coma cluster $(B \sim 2 \mu \mathrm{G})$, through measurements of the Faraday rotation effects in polarized sources seen through the cluster (Kim et al. 1990). Recently, Clarke et al. (1999) showed, in their statistical analysis of the rotation measure (RM) of polarized radio sources seen through 16 low redhsift $(z<0.1)$ clusters without radio halos, that the cluster magnetic fields are $B \sim 5-10 \mu \mathrm{G}$. Another method of directly measuring the cluster-wide $B$-field is from the detection of the inverse Compton (IC) scattered hard X-ray emission in excess of the thermal Bremsstrahlung emission. Recently, excess hard X-ray emission has been detected in the Coma cluster from Beppo-SAX, which gave a cluster $B$-field of $\sim 0.2 \mu \mathrm{G}$ (Fusco-Femiano et al. 1999). However, there are still debates over the origin of the detected hard X-ray excess, with suggestions of it being Bremsstrahlung radiation of supra-thermal electrons (e.g. Dogiel 2000).

So far no polarization has been detected in any known radio halo. In the case of 1E0657-56, the upper limit to the percentage polarization is $<20 \%$ on $55 \mathrm{kpc}$ scales at $1.3 \mathrm{GHz}$. The polarization upper limits at higher frequencies are in general poorer because of the steepness of the halo spectral indices. Radio relics, on the other hand, are found to be polarized. In 1E0657-56, the relic source is $\sim 10 \%$ polarized at $1.3 \mathrm{GHz}$ with mild depolarization. Similarly, in A2256 (Röttgering et al. 1997) and A3667 (Johnston-Hollitt private comm.), depolarization is only mild in the radio relics suggesting that relics are likely to be at the front of clusters. We deduce the B-field to be $>0.1 \mu \mathrm{G}$ from the average rotation measures of the relic. The rotation measure deduced from an extended, 
steep spectra, highly polarized radio source $(54 \%$ at $8.8 \mathrm{GHz})$, J0658-5559, also gave a lower limit of $0.2 \mu \mathrm{G}$ to the B-field of $1 \mathrm{E} 0657-56$ (Liang et al. in prep.).

\section{Model for Radio Halo Production}

The steep correlation $P_{1.4}-k T_{x}$ and the similarity in X-ray and radio halo morphology suggest that the electrons in the suprathermal high energy tail of the thermal gas distribution are likely to provide the seed particles for acceleration through mergers and turbulences to relativistic energies. These relativistic particles are then responsible for the production of the synchrotron emission. Dogiel (2000) pointed out that the hard X-ray excess (30-80 keV) observed in Coma by Beppo-SAX can be the result of Bremsstrahlung radiation from suprathermal electrons in the tail of the distorted Maxwellian spectrum. If there is in-situ acceleration, then the particle spectrum starts to deviate from the Maxwellian even below the injection energy due to Coulomb collisions. In this model, the only free parameters are the acceleration parameter and the particle production spectral index. Here we examine if the non-thermal tail of the Maxwellian can produce sufficient radio emission to explain the halo in Coma. The spectral index of the halo fixes the particle production spectral index and consequently the acceleration parameter given the observed hard X-ray excess. A magnetic field of $B \sim 1.5 \mu \mathrm{G}$ is necessary to produce the observed radio halo flux density (Dogiel \& Liang in prep.). In comparison, the magnetic field deduced from Faraday rotation gives $B<6 h_{50}^{1 / 2}, 3$ on scales $<1 \mathrm{kpc}$ with a large scale $(200 \mathrm{kpc})$ average magnetic field of $B \sim 0.2 \mu \mathrm{G}$ (Feretti et al. 1995); and the equipartition B-field is $\sim 0.9 \mu \mathrm{G}$. Hence, the model successfully fits the hard X-ray excess and the radio halo emission for the Coma cluster with a value of the cluster magnetic field consistent with current observations. Note that in this model, the IC radiation in the $30-80 \mathrm{keV}$ range is orders of magnitude smaller than the Bremsstrahlung radiation.

Acknowledgments. I thank my collaborators V. Dogiel, R. Hunstead, R. Ekers, M. Birkinshaw, P. Andreani, P. Shaver, I. Hook, W. Couch \& E. Falco for valuable contributions; and Prof. R. Buonanno for hospitality at the OAR.

\section{References}

Clarke, T., Kronberg, P. \& Böhringer, H. 1999, in Diffuse Thermal and Relativistic Plasma in Galaxy Clusters, eds. H. Böhringer et al., 82

Dogiel, V. 2000, A\&A, 357, 66

Feretti, L., Dallacasa, D., Giovannini, G. \& Tagliani, A. 1995 A\&A302, 680

Fusco-Femiano, R., et al. 1999, ApJ, 513, L21

Giovannini, G., Tordi, M. \& Feretti, L. 1999, New Astronomy 4, 141

Kempner, J.C. \& Sarazin, C.L. 2000, ApJ, in press

Kim, K.-T., Kronberg, P., Dewdney, P., \& Landecker, T. 1990, ApJ, 355, 29

Liang, H., Hunstead, R.W., Birkinshaw, M. \& Andreani, P. 2000, ApJ, in press

Röttgering, H., Wieringa, M., Hunstead, R. \& Ekers, R. 1997, MNRAS, 290, 577 